\title{
CACNA1C, schizophrenia and major depressive disorder in the Han Chinese population
}

Kuanjun He, ${ }^{*}$ Zhiguo An, Qingzhong Wang, Tao Li, Zhiqiang Li, Jianhua Chen, Wenjin Li, Ti Wang, Jue Ji, Guoyin Feng, He Lin, Qizhong Yi and Yongyong Shi

\section{Background}

Common psychiatric disorders are highly heritable, indicating that genetic factors play an important role in their aetiology. The CACNA1C gene, which codes for subunit alpha-1C of the Cav1.2 voltage-dependent L-type calcium channel, has been consistently found to be the shared risk gene for several kinds of mental disorder.

\begin{abstract}
Aims
To investigate whether CACNA1C is a susceptibility gene for schizophrenia and major depressive disorder in the Han chinese population.
\end{abstract}

\section{Method}

We carried out a case-control study of 1235 patients with schizophrenia, 1045 with major depressive disorder and 1235 healthy controls. A tag single nucleotide polymorphism (SNP) rs1006737 along with another 10 tag SNPS in the CACNA1C gene were genotyped in all samples.

\section{Results}

We found that rs1006737 was associated with both schizophrenia $\left(P_{\text {allele }}=0.0014, P_{\text {genotype }}=0.006\right.$, odds ratio $(\mathrm{OR})=1.384,95 \% \mathrm{Cl} 1.134-1.690)$ and major depressive disorder $\left(P_{\text {allele }}=0.0007, P_{\text {genotype }}=0.003, \mathrm{OR}=1.425,95 \% \mathrm{Cl}\right.$ 1.160-1.752)

\section{Conclusions}

Our findings support CACNA1C being a risk gene for both schizophrenia and major depressive disorder in the Han chinese population.

\section{Declaration of interest}

None.
As leading causes of morbidity that require significant long-term medical and social care, mental disorders have increasingly attracted attention in recent years. Schizophrenia, major depressive disorder and bipolar disorder are the three major disorders. Numerous family, twin and adoption studies confirmed that genetic factors play an important role in these mental disorders. ${ }^{1}$ Some of the underlying genetic risk factors for these three disorders have been identified in genetic association studies. Although the findings from these studies have often been inconsistent and have not been validated in different populations, some encouraging results have emerged recently. The CACNA1C gene has been found to be a risk gene for several major psychiatric disorders and has been validated in different populations. ${ }^{2-9}$ This gene is located at $12 \mathrm{p} 13.3$, spanning an approximately $645 \mathrm{~kb}$ genomic region, consists of 56 exons and encodes the alpha-1C subunit of the L-type voltage-dependent calcium channel Cav1.2. Cav1.2 couples transient increase of membrane permeability for calcium-causing cell-membrane depolarisation, leading to activated intracellular gene transcription and plays an important role in dendritic development, neuronal survival, synaptic plasticity, memory formation, learning and behaviour. ${ }^{10-13}$ The CACNA1C gene is widely expressed in the cardiovascular system and the entire nervous system, especially hippocampus and thalamus of brain. ${ }^{14,15}$ Genome-wide association studies (GWASs) have detected the single nucleotide polymorphism (SNP) rs1006737 in intron of the CACNA1C gene as a shared risk factor for schizophrenia, bipolar disorder and major depressive disorder in the White population. ${ }^{4,16}$ However, few genetic study of the CACNA1C gene have been carried out in the Han Chinese population. To investigate whether CACNA1C is associated with schizophrenia and major depressive disorder in the Han Chinese population, we genotyped rs1006737 along with ten other SNPs (online Table DS1) in 1235 people with

*These authors contributed equally to the work. schizophrenia, 1045 with major depressive disorder and 1235 controls of Han Chinese origin.

\section{Method}

\section{Participants}

Our sample set consists of 1235 people with schizophrenia (805 males and 430 females), 1045 participants with major depressive disorder (729 males and 316 females) and 1235 normal controls (665 males and 570 females) recruited from the Han Chinese population. All of the participants in our study were unrelated, living in Shanghai, China, and were of Shanghai origin. Patients were out-patients or in-patients whose condition was stable and were interviewed by two independent psychiatrists and diagnosed strictly according to DSM-IV criteria. ${ }^{17}$ All participants gave informed consent, the details of which had been reviewed and approved by the local ethical committee. Controls were randomly selected from the general population in Shanghai.

The mean age of individuals in the schizophrenia group was 36.4 years (s.d. =9.0). All of the participants with schizophrenia had paranoid schizophrenia and no lifetime history of an episode of mania or depression. The mean age of individuals in the major depressive disorder group was 34.4 years $($ s.d. $=12.1)$. They were carefully selected on the basis that all of them had experienced at least two distinct major depressive disorder episodes and displayed no signs of bipolar disorder during the 2-year period after the onset of depression. The mean age of individuals in the control group was 30.6 years $($ s.d. $=11.4)$. All controls were randomly selected from the general public of the Han Chinese population. Volunteers who replied to a written invitation completed an evaluation of their medical history, with supplementary questions about psychosis and other major complex diseases. Before collecting their blood, a face-to-face interview was conducted that included a physical examination (height, weight, blood pressure, etc.). 


\section{Genotyping}

Genomic DNA was prepared from peripheral blood samples of participants using the QuickGene DNA whole blood kit L protocol. The tag SNP selection was performed using haploview software, with pair-wise tagging, $r^{2} \geqslant 0.5$ and minor allele frequency $(\mathrm{MAF}) \geqslant 0.05 .{ }^{18,19}$ Details of all 11 SNPs genotyped in the CACNA1C gene can be found in Table DS1. All SNPs were genotyped using TaqMan SNP Genotyping Assays on a Fludigm EP1 platform. All probes were designed and synthesised by Life Technology. The criteria for excluding poorly performing samples and SNPs were determined by the genotype calls of each sample with a call-rate better than $96.5 \%$ (online Tables DS2 and DS3).

\section{Statistical analysis}

All the parameter calculations, including allele and genotype frequencies, and Hardy-Weinberg equilibrium analysis were carried out online using a SHEsis platform on Windows 7 (http:// analysis2. bio-X.cn/myAnalysis.php). ${ }^{20,21}$ All tests were two-tailed and statistical significance was assumed at the threshold of 0.05 .

\section{Population-stratification analysis}

To avoid the false-positive association caused by potential population stratification, we performed the stratification analysis on STRUCTURE software on Windows 7 (version 2.3.4, http:// pritch.bsd.uchicago. edu/structure.html). ${ }^{22-25}$ We used genotype data from 79 random SNPs to undertake population-stratification analysis on the third-stage sample set. We obtained data about these 79 SNPs from 522 HapMap samples, 174 samples from Utah residents with ancestry from northern and western Europe in the United States (CEU), 209 samples from Yoruba in Ibadan, Nigeria (YRI), and 139 samples from Han Chinese in Beijing (CHB) (HapMap public release 28 at http://hapmap.ncbi.nlm.nih.gov/ cgi-perl/gbrowse/hapmap28_B36/). ${ }^{26}$ The software assumes that there were $K$ populations ( $K$ is the number of assumed populations) in the data-set and then tries to find the distinct populations using the genotype data. Taking into consideration the immigration and geographical genetic isolation and the fact that the samples of the two disorders were both recruited from the same population, we applied the admixture model and correlated-frequencies model, with a burn-in length of 10000 and MCMC (Markov chain Monte Carlo) repeats of 10000 . To make sure the results were consistent, we ran the program several times at each $K$ from two to seven.

\section{Results}

We found that rs1006737 was positively associated with both schizophrenia (rs1006737: $P_{\text {allele }}=0.0014, P_{\text {genotype }}=0.006$, odds ratio $(\mathrm{OR})=1.384,95 \% \mathrm{CI} 1.134-1.690)$ and major depressive disorder $\left(\right.$ rs 1006737: $P_{\text {allele }}=0.0007, P_{\text {genotype }}=0.003, \mathrm{OR}=1.425$, 95\% CI 1.160-1.752) (online Tables DS4 and DS5). Moreover, after Bonferroni multiple tests correction, rs1006737 was still significantly associated with both disorders (online Tables DS4 and DS5).

Online Fig. DS1 shows the results of population-stratification analysis, which is the triangle chart of $K=3$. Each angle represents a possible independent ancestry and the different coloured dots represent the individuals in assumed population components. When $K=3$, the results described by the triangle chart are best. The combined population of CEU, CHB and YRI displayed a clear stratified pattern (Fig. DS1a). Our samples of the two disorders and controls distributed evenly in the triangle, which indicates that there was no obvious significant stratification in the population (Fig. DS1b). When $K$ ranged from two to seven, the results were consistent with each other. Taken together, we can therefore conclude that our positive results before correction were unlikely to have been caused by population stratification. (See online supplement DS1 for additional results.)

\section{Discussion}

\section{Findings relating to the CACNA1C gene}

Schizophrenia, major depressive disorder and bipolar disorder are three severe mental disorders. They not only affect individuals and their families but also challenge society and health services worldwide. A high degree of heritability has indicated that genetic factors play an important role in their aetiologies. The CACNA1C gene was reported to have a nominal association with bipolar disorder in a family-based association study. ${ }^{27}$ Combined with data from the Wellcome Trust Case-Control Consortium GWAS, ${ }^{28}$ Sklar et al reported that rs1006737, within the CACNA1C gene, was associated with bipolar disorder $\left(P=1 \times 10^{-4}\right)$ in a GWAS of 1461 people with bipolar disorder and 2008 controls. ${ }^{9}$ A subsequent study with a larger sample size confirmed that rs1006737, in CACNA1C, was associated with bipolar disorder $\left(P=7.0 \times 10^{-8}, \quad \mathrm{OR}=1.181\right) \quad$ in a White population. $^{2}$ The CACNA1C gene has also been found to be associated with other psychiatric disorders. Genetic studies have discovered that the genotype of CACNA1C was also associated with schizophrenia ${ }^{5,29}$ and major depressive disorder. ${ }^{7,30}$ Green et al reported in a GWAS that the risk SNP (rs1006737) for bipolar disorder was also a conferred risk for schizophrenia and early recurrent major depressive disorder. ${ }^{3}$ A meta-analysis of two separate GWASs of people with bipolar disorder and major depressive disorder revealed that rs 1006737 reached genome-wide significance after combining bipolar with unipolar mood disorders. ${ }^{4}$ The consistent findings in genetic studies indicates that the CACNA1C gene belongs to a class of shared susceptibility factors for major psychiatric disorders and has likely played an important role in the pathogenesis of them.

Nevertheless, the mechanisms underlying how genetic changes in CACNA1C modify risk for developing psychiatric disorders are still unclear. This gene encodes the alpha-1C subunit of the L-type voltage-dependent calcium channel Cav1.2. Calcium channels are involved in various aspects of neuronal development and in the establishment of maintenance of connectivity during development and throughout adulthood. ${ }^{31}$ Previous studies of people with bipolar disorder have consistently reported elevated basal and stimulated intracellular calcium levels in peripheral blood cells. $^{32,33}$ The evidences suggested that calcium signalling may play a role in bipolar disorder. Cav1.2 participates in the proper function of numerous neurological circuits in the hippocampus, amygdala and mesolimbic reward and motivation systems. ${ }^{34,35}$ These are strongly implicated in psychiatric disease pathophysiology.

The CACNA1C gene has also been found to be expressed in many tissues in the human body across various developmental stages. ${ }^{36}$ And, it is widely expressed in the brain, especially hippocampus and thalamus. ${ }^{15}$ The relevance of CACNA1C to bipolar disorder was supported by the observed downregulation of messenger (m)RNA transcripts in mouse brain in response to lithium. ${ }^{37}$ Notably, a missense mutation in CACNA1C can cause Timothy syndrome, which is characterised by multi-organ dysfunction such as cardiac arrhythmias and cognitive abnormalities. $^{38}$ The heterozygous knockout adult mice of CACNA1C were found expressing decreased Cav1.2 protein levels and L-type calcium channel current, and protecting against depression-like phenotypes. ${ }^{39}$ A larger effect-size study clearly 
indicated that genetic variant at SNP rs1006737 was associated with changes in brain structure and function in normal controls. ${ }^{40}$ Genetic variation at rs1006737 may be involved in modulating gene expression, whereas the risk allele of rs1006737 is associated with increased expression levels of CACNA1C. ${ }^{29}$ Three studies found the rs 1006737 risk allele was associated with increased brain grey matter, either total grey matter volume or in specific brain regions in healthy controls. ${ }^{41-43}$

In our study, the main finding was a significant association between the CACNA1C gene and both schizophrenia and major depressive disorder in the Han Chinese population. We have confirmed that rs1006737 in CACNA1C was significantly associated with schizophrenia $\left(P_{\text {allele }}=0.0014, P_{\text {genotype }}=0.006, \mathrm{OR}=1.384\right.$, MAF $=0.101)$ and major depressive disorder $\left(P_{\text {allele }}=0.0007\right.$, $\left.P_{\text {genotype }}=0.003, \mathrm{OR}=1.425, \mathrm{MAF}=0.103\right)$ in a case-control study with a large sample size in the Han Chinese population. Even when Bonferroni correction (it is considered the most conservative method) was used, we still found that rs1006737 was associated with schizophrenia $\left(P_{\text {allele }}=0.028\right)$ and major depressive disorder $\left(P_{\text {allele }}=0.014\right)$. At the same time, we found another SNP (rs2239015) was significantly associated with schizophrenia (after Bonferroni correction: $P_{\text {allele }}=0.006, P_{\text {genotype }}=0.030$, $\mathrm{OR}=1.249, \mathrm{MAF}=0.355)($ Table DS4).

Nyegaard et al reported that rs1006737 was associated with schizophrenia $(P=0.015, \mathrm{OR}=1.16, \mathrm{MAF}=0.361)$ in a casecontrol study of 976 people with schizophrenia and 1489 healthy controls of European origin. ${ }^{5}$ Green et al have reported that rs1006737 is associated with major depressive disorder $(P=0.013, \mathrm{OR}=1.15, \mathrm{MAF}=0.363)$ in recurrent major depressive disorder $(n=1196)$ and UK non-psychiatric comparison groups $(n=15316){ }^{3}$ Comparing our results with theirs, the MAFs for the SNPs were quite different but the ORs are all in the same direction (for schizophrenia, OR: 1.384 v. 1.16, MAF: $0.101 v$. 0.361 ; for major depressive disorder, OR 1.425 v. 1.15, MAF 0.103 v. 0.363).

Avoiding the potential influence of population stratification in our samples was important. We therefore used the software STRUCTURE 2.3.4 and data from 79 additional random SNPs dispersed on different chromosomes to analyse the potential population stratification in our samples. We did not detect any population stratification, and therefore, the results could not be affected by this confounding factor.

\section{Future directions for research}

Given rs1006737 and rs2239015 were both located in introns of CACNA1C, they were not expected to directly interfere with properties relating to the structure and function of Cav1.2. We know that intronic variation is likely to be involved in regulating gene expression and that extensive alternative splicing exists in the CACNA1C transcripts. It may potentially generate thousands of splice variants. Now, it is important to confirm the consequences of changes in splice variation of CACNA1C on neurophysiology. If we can find relevant answers, it might be very helpful for deciding novel therapeutic targets and approaches. In addition, it is also necessary to test its association with bipolar disorder in a large sample of individuals of Han Chinese origin in the future.

\section{Funding}

This work was supported by the Natural Science Foundation of China $(81130022,81272302$, 31000553, 81121001), the National 863 project (2012AA02A515), the 973 Program (2010CB529600), Program for Changjiang Scholars and Innovative Research Team in University (IRT1025), the Foundation for the Author of National Excellent Doctoral Dissertation of China (201026), Shanghai Rising-Star Program (12QA1401900) and 'Shu Dissertation of China (201026), Shanghai Rising-Star Program (12QA1401900) and 'Shu
Guang' project supported by Shanghai Municipal Education Commission and Shanghai Education Development Foundation (12SG17).

\section{Acknowledgements}

We are grateful to all patients and healthy controls participating in this study, as well as to the psychiatrists for their help in the recruitment and identification of patients with schizophrenia and major depressive disorder.

Kuanjun He, PhD, Bio-X Institutes, Key Laboratory for the Genetics of Developmental and Neuropsychiatric Disorders (Ministry of Education), Shanghai Jiao Tong University, Shanghai and College of Life Science, Inner Mongolia University for Nationalities, Tongliao, Inner Mongolia; Zhiguo An, MS, Department of Psychiatry, the First Teaching Hospital of Xinjiang Medical University, Urumqi; Qingzhong Wang, PhD, Tao Li, PhD, Zhiqiang Li, PhD, Bio-X Institutes, Key Laboratory for the Genetics of Developmental and Neuropsychiatric Disorders (Ministry of Education), Shanghai Jiao Tong University, Shanghai; Jianhua Chen, PhD, Bio-X Institutes, Key Laboratory for the Genetics of Developmental and Neuropsychiatric Disorders (Ministry of Education), Shanghai Jiao Tong University, Shanghai and Schizophrenia Program, Shanghai Mental Health Center, Shanghai Jiao Tong University School of Medicine, Shanghai; Wenjin Li, PhD, Ti Wang, PhD, Jue Ji, BM, Bio-X Institutes, Key Laboratory for the Genetics of Developmental and Neuropsychiatric Disorders (Ministry of Education), Shanghai Jiao Tong University, Shanghai and College of Life Science, Inner Mongolia University for Nationalities, Tongliao, Inner Mongolia; Guoyin Feng, BM, Schizophrenia Program, Shanghai Mental Health Center, Shanghai Jiao Tong University School of Medicine, Shanghai; He Lin, PhD, Bio-X Institutes, Key Laboratory for the Genetics of Developmental and Neuropsychiatric Disorders (Ministry of Education), Shanghai Jiao Tong University, Shanghai; Qizhong Yi, MD, PhD,

Department of Psychiatry, the First Teaching Hospital of Xinjiang Medical University, Urumqi; Yongyong Shi, PhD, Bio-X Institutes, Key Laboratory for the Genetics of Developmental and Neuropsychiatric Disorders (Ministry of Education), Shanghai Jiao Tong University, Shanghai, Shanghai Changning Mental Health Center, Shanghai and Institute of Neuropsychiatric Science and Systems Biological Medicine, Shanghai Jiao Tong University, Shanghai, People's Republic of China

Correspondence: Yongyong Shi or Qizhong Yi or Lin He, Bio-X Institutes, Shanghai Jiao Tong University, The Central Little White House, 1954 Huashan Road, Shanghai 200030 or Department of Psychiatry, the First Teaching Hospital of Xinjiang Medical University, Urumqi 830002, People's Republic of China. Tel./fax: +86 2162822491. Email: shiyongyong@gmail.com (Y. Shi) or 13079911689@126.com (Q. Yi) or helinhelin@gmail.com (L. He).

First received 27 Jan 2013, final revision 10 May 2013, accepted 5 Jun 2013

\section{References}

1 Craddock N, O'Donovan MC, Owen MJ. The genetics of schizophrenia and bipolar disorder: dissecting psychosis. J Med Genet 2005; 42: 193-204.

2 Ferreira MA, O'Donovan MC, Meng YA, Jones IR, Ruderfer DM, Jones L, et al. Collaborative genome-wide association analysis supports a role for ANK3 and CACNA1C in bipolar disorder. Nat Genet 2008; 40: 1056-8.

3 Green EK, Grozeva D, Jones I, Jones L, Kirov G, Caesar S, et al. The bipolar disorder risk allele at CACNA1C also confers risk of recurrent major depression and of schizophrenia. Mol Psychiatry 2010; 15: 1016-22.

4 Liu Y, Blackwood DH, Caesar S, de Geus EJ, Farmer A, Ferreira MA, et al. Meta-analysis of genome-wide association data of bipolar disorder and major depressive disorder. Mol Psychiatry 2011; 16: 2-4.

5 Nyegaard M, Demontis D, Foldager L, Hedemand A, Flint TJ, Sorensen KM, et al. CACNA1C (rs1006737) is associated with schizophrenia. Mol Psychiatry 2010; 15: 119-21.

6 Ripke S, Sanders A, Kendler K, Levinson D, Sklar P, Holmans P, et al. Genome-wide association study identifies five new schizophrenia loci. Nat Genet 2011; 43: 969-76.

7 Shi J, Potash JB, Knowles JA, Weissman MM, Coryell W, Scheftner WA, et al. Genome-wide association study of recurrent early-onset major depressive disorder. Mol Psychiatry 2011; 16: 193-201.

8 Sklar P, Ripke S, Scott L, Andreassen OA, Cichon S, Craddock N, et al. Large-scale genome-wide association analysis of bipolar disorder identifies a new susceptibility locus near ODZ4. Nat Genet 2011; 43: 977-83.

9 Sklar P, Smoller JW, Fan J, Ferreira MA, Perlis RH, Chambert K, et al. Whole-genome association study of bipolar disorder. Mol Psychiatry 2008; 13: $558-69$.

10 Moosmang S, Haider $\mathrm{N}$, Klugbauer $\mathrm{N}$, Adelsberger $\mathrm{H}$, Langwieser $\mathrm{N}$, Muller J, et al. Role of hippocampal Cav1.2 $\mathrm{Ca}^{2+}$ channels in NMDA receptorindependent synaptic plasticity and spatial memory. J Neurosci 2005; 25 9883-92.

11 Shibasaki M, Kurokawa K, Ohkuma S. Upregulation of L-type Ca(v)1 channels in the development of psychological dependence. Synapse 2010; 64: 440-4.

12 White JA, McKinney BC, John MC, Powers PA, Kamp TJ, Murphy GG. Conditional forebrain deletion of the L-type calcium channel Ca V 1.2 disrupts remote spatial memories in mice. Learn Mem 2008; 15: 1-5. 
13 Woodside BL, Borroni AM, Hammonds MD, Teyler TJ. NMDA receptors and voltage-dependent calcium channels mediate different aspects of acquisition and retention of a spatial memory task. Neurobiol Learn Mem 2004; 81 $105-14$.

14 Narayanan D, Xi Q, Pfeffer LM, Jaggar JH. Mitochondria control functional CaV1.2 expression in smooth muscle cells of cerebral arteries. Circ Res 2010; 107: $631-41$.

15 Striessnig J, Koschak A, Sinnegger-Brauns MJ, Hetzenauer A, Nguyen NK Busquet $\mathrm{P}$, et al. Role of voltage-gated L-type $\mathrm{Ca}^{2+}$ channel isoforms for brain function. Biochem Soc Trans 2006; 34: 903-9.

16 Curtis $D$, Vine $A E$, McQuillin A, Bass NJ, Pereira A, Kandaswamy R, et al. Case-case genome-wide association analysis shows markers differentially associated with schizophrenia and bipolar disorder and implicates calcium channel genes. Psychiatr Genet 2011; 21: 1-4.

17 American Psychiatric Association. Diagnostic and Statistical Manual of Mental Disorders (4th edn) (DSM-IV). APA, 1994.

18 de Bakker PI, Burtt NP, Graham RR, Guiducci C, Yelensky R, Drake JA et al. Transferability of tag SNPs in genetic association studies in multiple populations. Nat Genet 2006; 38: 1298-303.

19 de Bakker PI, Yelensky R, Pe'Er I, Gabriel SB, Daly MJ, Altshuler D. Efficiency and power in genetic association studies. Nat Genet 2005; 37 : 1217-23.

20 Li Z, Zhang Z, He Z, Tang W, Li T, Zeng Z, et al. A partition-ligationcombination-subdivision EM algorithm for haplotype inference with multiallelic markers: update of the SHEsis (http://analysis.bio-X.cn) Cell Res 2009; 19: 519-23.

21 Shi YY, He L. SHEsis, a powerful software platform for analyses of linkage disequilibrium, haplotype construction, and genetic association at polymorphism loci. Cell Res 2005; 15: 97-8.

22 Falush $D$, Stephens $M$, Pritchard JK. Inference of population structure using multilocus genotype data: dominant markers and null alleles. Mol Ecol Notes 2007; 7: 574-8.

23 Falush D, Stephens M, Pritchard JK. Inference of population structure using multilocus genotype data: linked loci and correlated allele frequencies. Genetics 2003; 164: 1567-87.

24 Pritchard JK, Stephens M, Donnelly P. Inference of population structure using multilocus genotype data. Genetics 2000; 155: 945-59.

25 Hubisz MJ, Falush D, Stephens M, Pritchard JK. Inferring weak population structure with the assistance of sample group information. Mol Ecol Resour 2009: 9: 1322-32.

26 International HapMap Consortium. The International HapMap Project. Nature 2003; 426: 789-96.

27 Sklar P, Gabriel SB, McInnis MG, Bennett P, Lim YM, Tsan G, et al. Familybased association study of 76 candidate genes in bipolar disorder: BDNF is potential risk locus. Brain-derived neutrophic factor. Mol Psychiatry 2002; 7 579-93.

28 Burton P, Clayton D, Cardon L, Craddock N, Deloukas P, Duncanson A, et al Genome-wide association study of 14,000 cases of seven common diseases and 3,000 shared controls. Nature 2007; 447: 661-78.
29 Bigos KL, Mattay VS, Callicott JH, Straub RE, Vakkalanka R, Kolachana B, et al. Genetic variation in CACNA1C affects brain circuitries related to mental illness. Arch Gen Psychiatry 2010; 67: 939-45.

30 Casamassima F, Huang J, Fava M, Sachs GS, Smoller JW, Cassano GB, et al. Phenotypic effects of a bipolar liability gene among individuals with major depressive disorder. Am J Med Genet B Neuropsychiatr Genet 2010; 153B: 303-9.

31 Spitzer NC. Electrical activity in early neuronal development. Nature 2006; 444: 707-12.

32 Emamghoreishi M, Schlichter L, Li PP, Parikh S, Sen J, Kamble A, et al. High intracellular calcium concentrations in transformed lymphoblasts from subjects with bipolar I disorder. Am J Psychiatry 1997; 154: 976-82.

33 Perova T, Wasserman MJ, Li PP, Warsh JJ. Hyperactive intracellular calcium dynamics in B lymphoblasts from patients with bipolar I disorder. Int $J$ Neuropsychopharmacol 2008; 11: 185-96.

34 Leitch B, Szostek A, Lin R, Shevtsova O. Subcellular distribution of L-type calcium channel subtypes in rat hippocampal neurons. Neuroscience 2009; 164: 641-57.

35 Nestler EJ, Carlezon WJ. The mesolimbic dopamine reward circuit in depression. Biol Psychiatry 2006; 59: 1151-9.

36 Pang L, Koren G, Wang Z, Nattel S. Tissue-specific expression of two human $\mathrm{Ca}(\mathrm{v}) 1.2$ isoforms under the control of distinct $5^{\prime}$ flanking regulatory elements. Febs Lett 2003: 546: 349-54.

37 McQuillin A, Rizig M, Gurling HM. A microarray gene expression study of the molecular pharmacology of lithium carbonate on mouse brain mRNA to understand the neurobiology of mood stabilization and treatment of bipolar affective disorder. Pharmacogenet Genomics 2007; 17: 605-17.

38 Splawski I, Timothy KW, Sharpe LM, Decher N, Kumar P, Bloise R, et al. $\mathrm{Ca}(\mathrm{V}) 1.2$ calcium channel dysfunction causes a multisystem disorder including arrhythmia and autism. Cell 2004; 119: 19-31.

39 Dao DT, Mahon PB, Cai X, Kovacsics CE, Blackwell RA, Arad M, et al. Mood disorder susceptibility gene CACNA1C modifies mood-related behaviors in mice and interacts with sex to influence behavior in mice and diagnosis in humans. Biol Psychiatry 2010; 68: 801-10.

40 Rose EJ, Donohoe G. Brain vs behavior: an effect size comparison of neuroimaging and cognitive studies of genetic risk for schizophrenia. Schizophr Bull 2013; 39: 518-26.

41 Kempton MJ, Ruberto G, Vassos E, Tatarelli R, Girardi P, Collier D, et al. Effects of the CACNA1C risk allele for bipolar disorder on cerebral gray matter volume in healthy individuals. Am J Psychiatry 2009; 166: 1413-4.

42 Perrier E, Pompei F, Ruberto G, Vassos E, Collier D, Frangou S. Initial evidence for the role of CACNA1C on subcortical brain morphology in patients with bipolar disorder. Eur Psychiatry 2011; 26: 135-7.

43 Wang F, McIntosh AM, He Y, Gelernter J, Blumberg HP. The association of genetic variation in CACNA1C with structure and function of a frontotemporal system. Bipolar Disord 2011; 13: 696-700. 